\title{
MedienPädagogik
}

Zeitschrift für Theorie und Praxis der Medienbildung

\section{Editorial: Theoriebildung in Mediendidaktik und Wissensmanagement}

Claudia de Witt und Michael Kerres

Das Internet trägt massgeblich zur Entstehung einer neuen Lern- und Wissenskultur bei und ist mittlerweile Gegenstand einer ganzen Reihe von Projekten in der Erziehungswissenschaft. So wird zum Beispiel der Frage nachgegangen, welche Möglichkeiten der Strukturierung und Gestaltung von Lernmaterialien am besten geeignet sind, um Lernprozesse anzuregen, wie die interpersonale Kommunikation im Internet durch didaktische Gestaltungsmassnahmen gefördert werden können oder wie die entfernte Betreuung von Lernenden über das Netz funktioniert. Zunehmend wird dabei auch der Übergang bedeutsam zwischen didaktisch aufbereiteten Lernangeboten, wie sie die Mediendidaktik traditionell beschäftigt, und dem Austausch von nicht didaktisch aufbereiteten Informationen in Organisationen, wie es im Bereich des Wissensmanagements diskutiert wird.

In diesen Projekten werden vielfach Detailfragen behandelt, und isoliert, ohne Bezug zu grundlegenden Theoriefragen untersucht. Es mangelt an einer genuin mediendidaktischen Theoriediskussion, die einen übergreifenden Bezugsrahmen aus erziehungswissenschaftlicher Perspektive liefert. In der Vergangenheit ist die Mediendidaktik von Seiten einer «kritischen» Medienpädagogik zeitweise skeptisch beäugt und in ihrer Existenz grundlegend infrage gestellt worden. In der aktuellen Diskussion wird gerne auf lerntheoretische Ansätze zurückgegriffen, die Lernen als aktiven Vorgang der individuellen und kooperativen Wissenskonstruktion begreifen. Doch Wissens- und Lerntheorien ersetzen keine mediendidaktische Modell- und Theoriebildung, die Perspektiven für eine Gestaltung zukünftiger Lernwelten liefert und dabei zum Beispiel sowohl bildungstheoretische Überlegungen als auch Konzepte des instructional design einbezieht.

In dem ersten Teil dieser Ausgabe geht es um theoretische Perspektiven der Mediendidaktik. So diskutiert der Artikel von Michael Kerres und Claudia de Witt den Beitrag der Positionen von Behaviorismus, Kognitivismus und Konstruktivismus zur theoretischen Fundierung der Mediendidaktik. Er geht auf Missverständnisse und Probleme in diesem Zusammenhang ein. Als alternative Sichtweise stellen die Autoren eine Interpretation des Pragmatismus von John Dewey vor, der von der Lernsituation ausgeht und einen methodisch orientierten Ansatz anbietet. Damit wird letztlich eine stärkere Verbindung von Theorien der Mediendidaktik und Medienbildung angestrebt.

Auch Stefan Krause und Rolf-Dieter Kortmann ziehen die Lerntheorien zur Diskussion der medienbasierten Wissensaneignung heran, sie legen ihren Schwerpunkt aber auf die Frage nach der aktuell diskutierten Problematik der Standardisierung

de Witt, Claudia, und Michael Kerres. 2002. «Editorial: Theoriebildung in Mediendidaktik und Wissensmanagement». MedienPädagogik 6, (OKtober), i-ii. https://doi.org/10.21240/mpaed/06/2002.10.25.X. 
bzw. Standardisierbarkeit von medial aufbereiteten Lerninhalten. Sie beschreiben, wie der Austausch von Inhalten perspektivisch nicht mehr über Datenformate, sondern durch Lernobjekte stattfindet, und diskutieren anhand eines Beispiels die - problematischen - Implikationen für die Mediendidaktik.

Einen anderen Impuls gibt der Beitrag von Wolfgang Müskens und Isabel Müskens. Sie verstehen «Provokationen» als methodisches Element einer Didaktik internetgestützter Lernarrangements. "Provoziert» werden soll der Lernende durch einstellungskonträres Material, das letztlich zu einer höheren Individualisierung des Lernens und einer stärkeren Interaktion zwischen Lehrenden und Lernenden führen soll. Die theoretische Begründung solcher provokativen Elemente in einem didaktischen Konzept findet sich im Kompetenzbegriff und in der Systemtheorie, aber auch in psychologischen Ansätzen.

In dem zweiten Teil dieser Ausgabe der Online-Zeitschrift geht es insbesondere um den Zusammenhang von Mediendidaktik und Wissensmanagement. Für Gabi Reinmann-Rothmeier handelt es sich bei Wissensmanagement und Mediendidaktik bislang um ein dialektisches Paar. Während Wissensmanagement eher ein Thema für die Betriebswirtschaft war, wurde die Mediendidaktik als eine (Teil-)Disziplin der Medienpädagogik verstanden. Durch E-Learning wachsen diese Bereiche zusehends zusammen. Anhand von zwei Orientierungsmodellen aus pädagogisch-psychologischer Sicht werden die Berührungspunkte von Mediendidaktik und Wissensmanagement herausgearbeitet.

Welche Anregungen lassen sich aus der Verbindung von Wissensmanagement und E-Learning gewinnen? Zur Beantwortung dieser Frage stellt Andrea Back ein ELearning-Referenzmodell vor. Es formuliert eine Basis für den Erwerb von Medienkompetenz und beinhaltet gleichzeitig ein Untersuchungsinstrument für die Beurteilung des Einsatzes von Wissensmanagement für E-learning.

Andreas Breiter fragt nach dem Erfolg von Wissensmanagementsystemen mit neuen Medien in der Schule. Er verdeutlicht seine Erfahrungen anhand der Entwicklung eines webbasierten Wissensmanagementsystems.

Wir wünschen Ihnen viel Spass beim Lesen und hoffen mit dieser Ausgabe eine Diskussion zur Theoriebildung in der Mediendidaktik anzuregen. 